\title{
Resonant Light-Induced Heating in Hybrid Cavity-Coupled 2D
}

\section{Transition Metal Dichalcogenides}

Hossein Taghinejad ${ }^{1}$, Mohammad Taghinejad ${ }^{1}$, Alexey Tarasov ${ }^{2}$, Meng-Yen Tsai ${ }^{2}$, Amir H. Hosseinnia $^{1}$, Hesam Moradinejad ${ }^{1}$, Philip M. Campbell ${ }^{2}$, Ali A. Eftekhar ${ }^{1}$, Eric M. Vogel ${ }^{2}$, Ali Adibi $^{1{ }^{*}}$

1: School of Electrical and Computer Engineering, Georgia Institute of Technology, 778 Atlantic Drive NW, Atlanta, GA 30332, USA

2: School of Materials Science and Engineering, Georgia Institute of Technology, 771 Ferst Drive NW, Atlanta, GA 30332, USA

*: Corresponding Author: ali.adibi@ece.gatech.edu

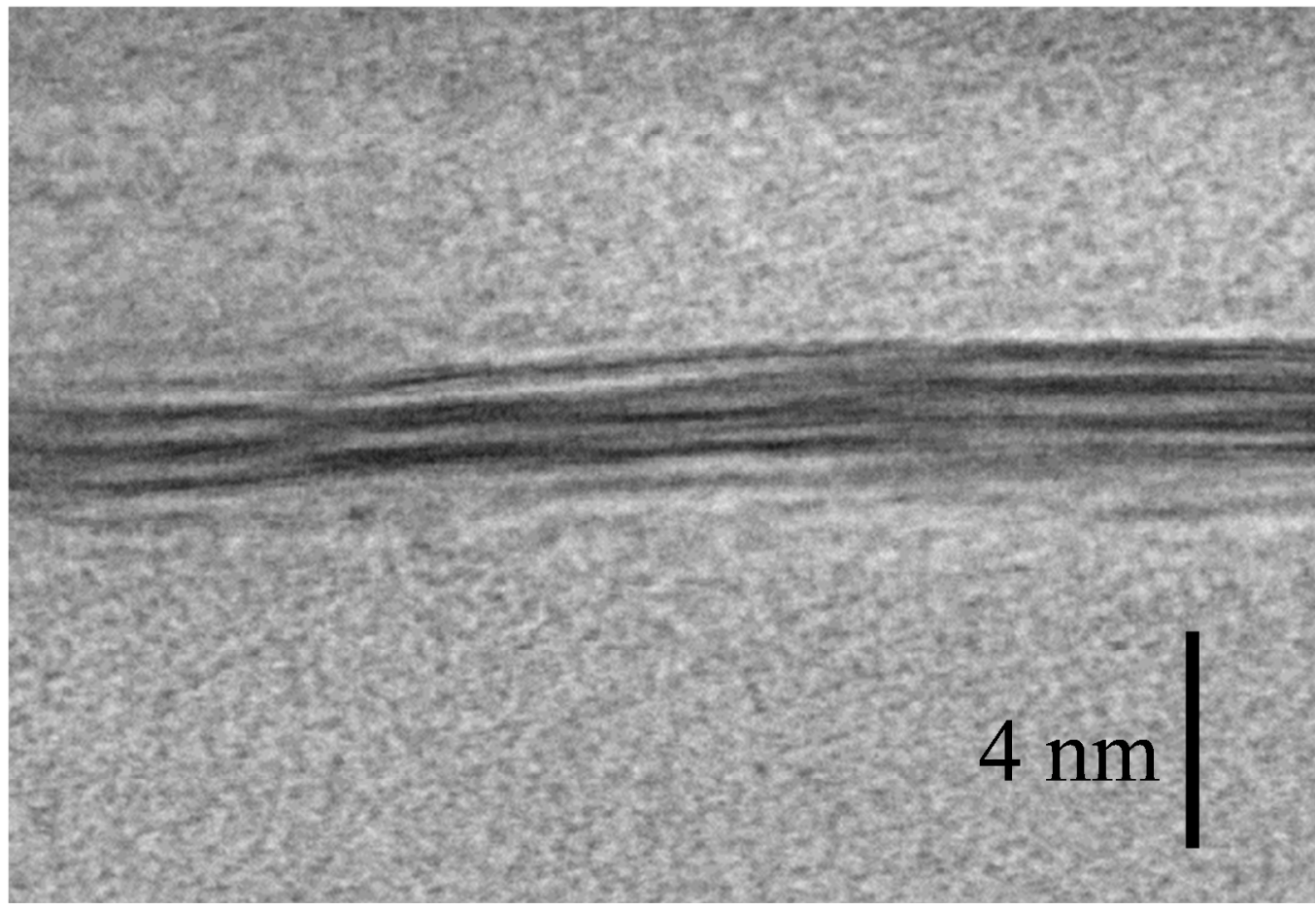

Figure S1: Cross-sectional transmission electron microscopy (TEM) image showing stack of three individual $\mathrm{MoS}_{2}$ layers (Mo layers appear darker than $\mathrm{S}$ layers in the image). 

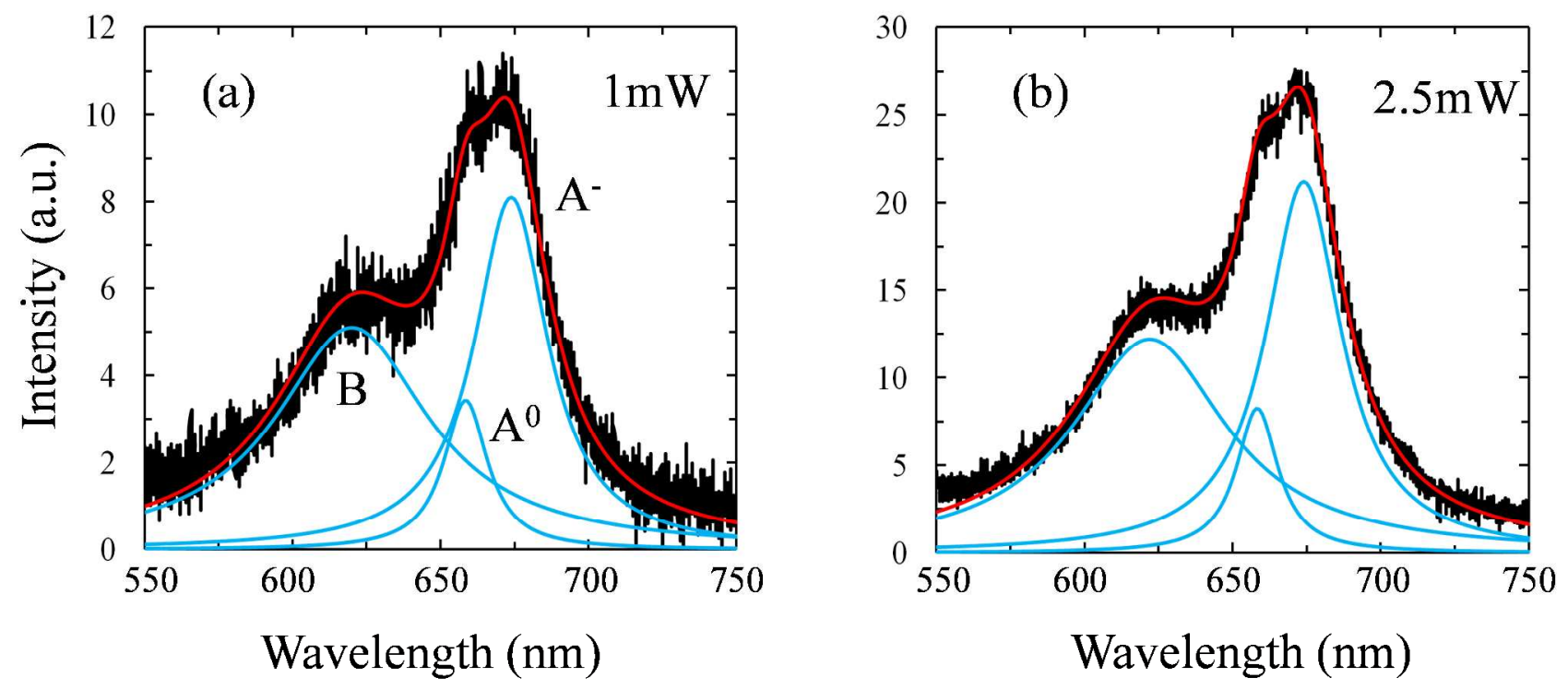

Figure S2: Photoluminescence (PL) spectra of trilayer $\mathrm{MoS}_{2}$ film at (a) $1 \mathrm{~mW}$ and (b) $2.5 \mathrm{~mW}$ excitation laser powers. Black lines represent experimental data and red lines are Lorentzian fits. Blue lines are components of the Lorentzian fits representative of luminescence from B excitons, neutral A excitons $\left(\mathrm{A}^{0}\right)$, and negatively charged $\mathrm{A}$ excitons $\left(\mathrm{A}^{-}\right)$as marked in part (a). The existence of the negatively charged exciton (the so-called trion) points to the n-type nature of the $\mathrm{MoS}_{2}$ film, an effect that is often observable upon wet transfer of $\mathrm{MoS}_{2}$ films. 

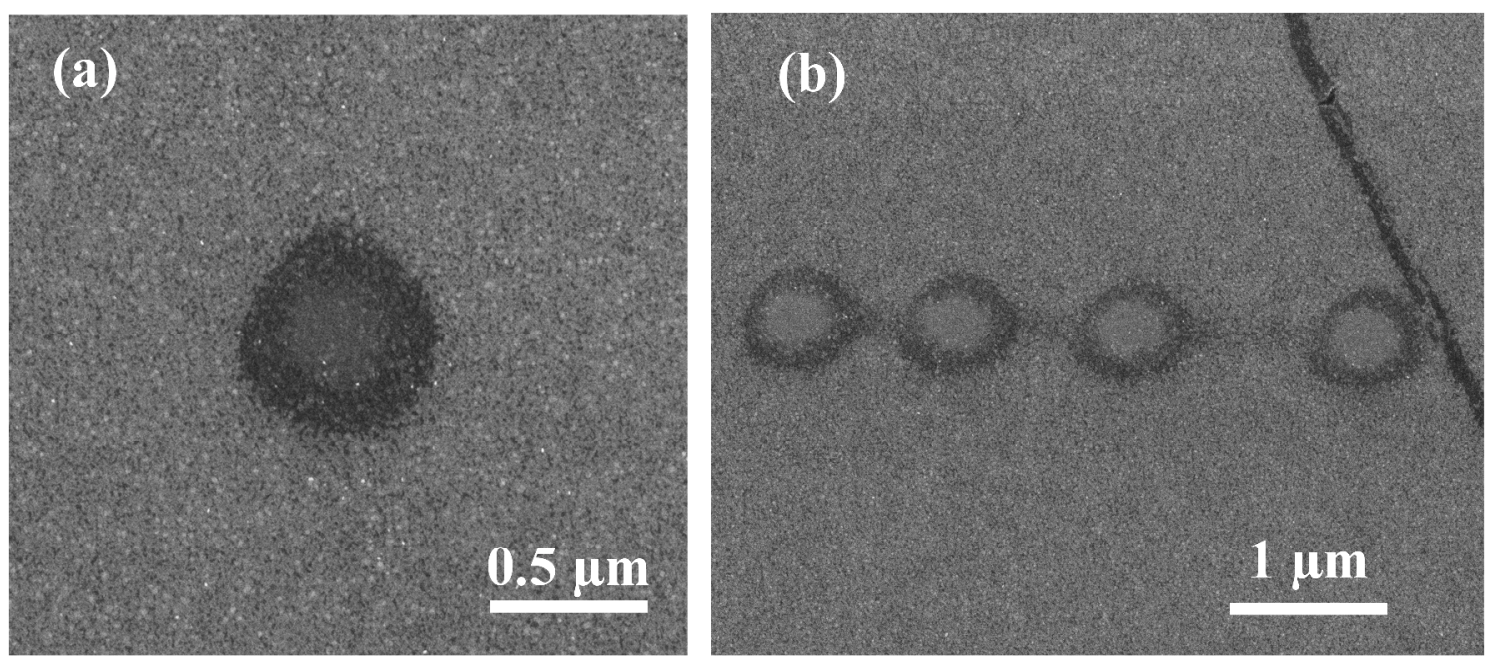

Figure S3: Scanning electron microscopy (SEM) image of permanent damage to the $\mathrm{MoS}_{2}$-on-FP sample. (a) Damaging of the $\mathrm{MoS}_{2}$ film after exposure to $5 \mathrm{~mW}$ laser power can be inferred by observing that the permanent trace of the laser spot on the sample. The darker regions are the damaged regions because of the excess heating of the $\mathrm{MoS}_{2}$ film. Cavity length (i.e., $\mathrm{SiO}_{2}$ thickness) in this sample is $256 \mathrm{~nm}$. (b) To verify this effect, we displace the laser spot and expose the $\mathrm{MoS}_{2}$ to similar laser power of $5 \mathrm{~mW}$. As this SEM image depicts, same damaged pattern repeats on the surface. It is worth noting that the laser power threshold at which damaging happens changes by changing the cavity length. The stronger the light-MoS interaction is, the lower the laser threshold will be. 


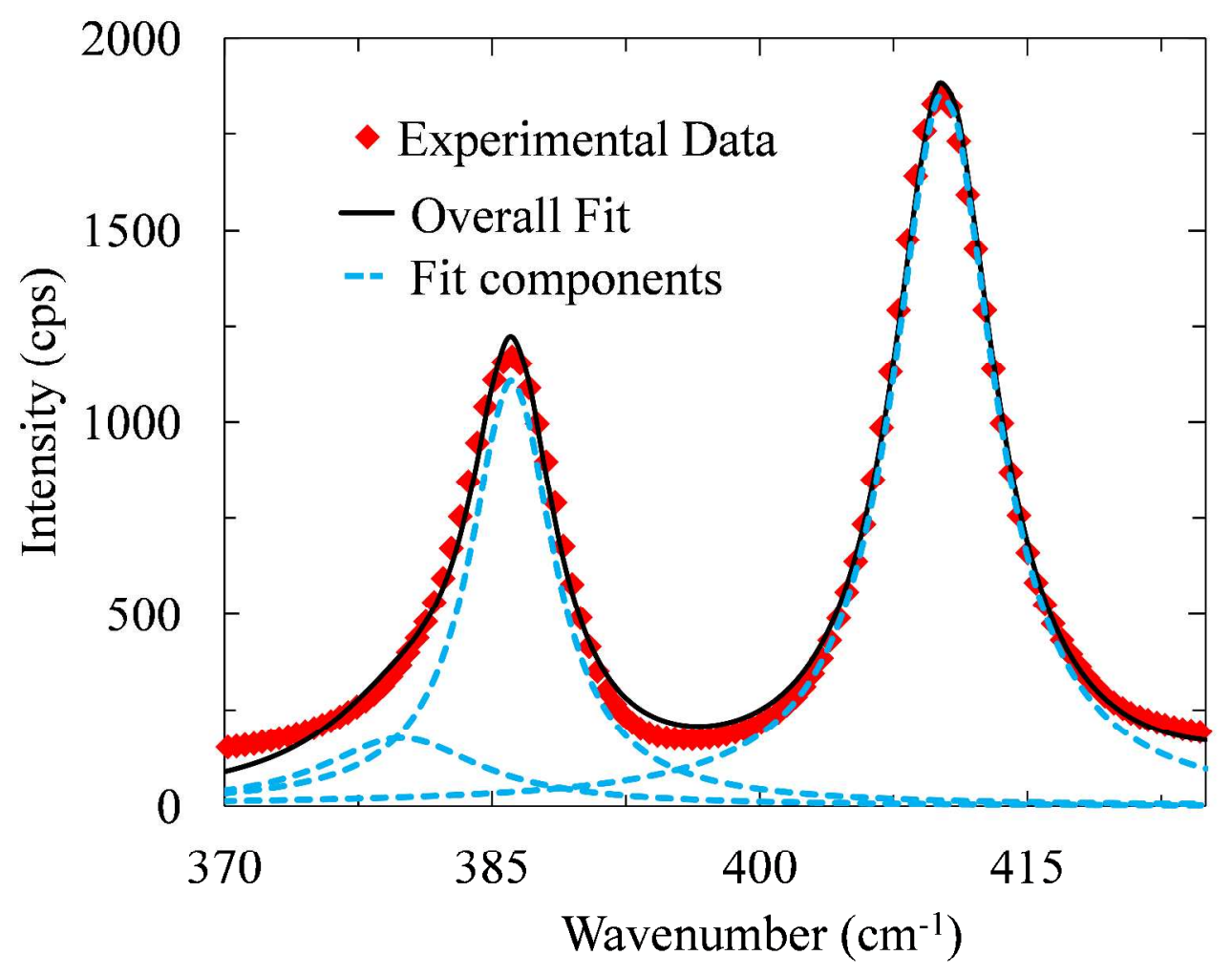

Figure S4: A sample Lorentzian fit (solid line) to the experimentally acquired Raman data (red squares) of the $\mathrm{MoS}_{2}$ film obtained at $3 \mathrm{~mW}$ laser power. The Raman spectrum is composed of three components (dashed lines): $A_{1 g}$ out-of-plane vibration at $410.3 \mathrm{~cm}^{-1}, \mathrm{E}_{2 \mathrm{~g}}^{1}$ in-plane vibration at $386.1 \mathrm{~cm}^{-1}$, and $\mathrm{E}_{2 \mathrm{~g}}^{1}$ conjugate vibration at $379.9 \mathrm{~cm}^{-1}[\mathrm{~S} 1]$. 
(a)

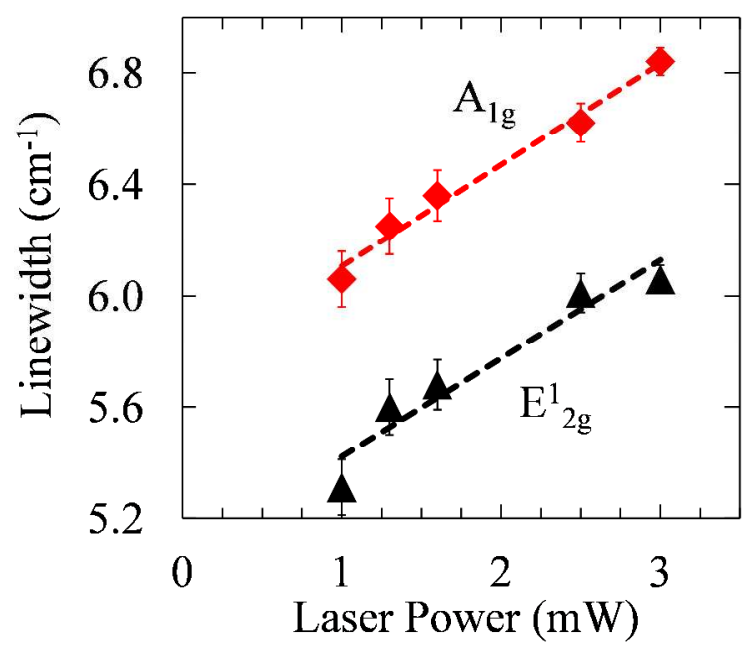

(b)

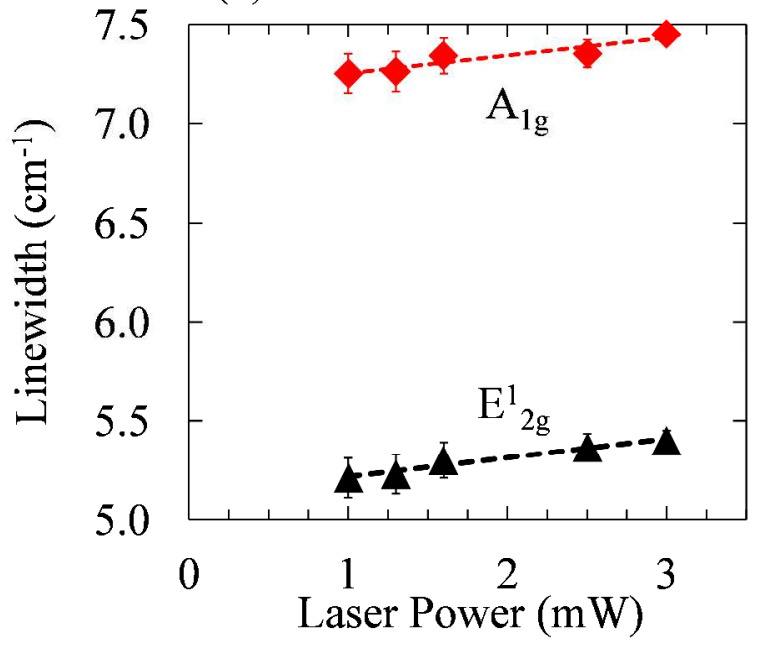

Figure S5: Linewidths of the $\mathrm{E}_{2 \mathrm{~g}}^{1}$ and $\mathrm{A}_{1 \mathrm{~g}}$ resonances of the (a) $\mathrm{MoS}_{2}$-on-FP and (b) $\mathrm{MoS}_{2}$-on-Si samples measured at different excitation laser powers. Increasing the laser power broadens the resonance spectra on both samples while a much stronger broadening is observed on the $\mathrm{MoS}_{2^{-}}$ on-FP sample. The FP-cavity length (i.e., $\mathrm{SiO}_{2}$ thickness) is $256 \mathrm{~nm}$ in $\mathrm{MoS}_{2}$-on-FP sample, corresponding to the sample used for the analysis of Figure 1 in the main text. The dashed lines are linear fits to the experimental data (square and triangle symbols). 


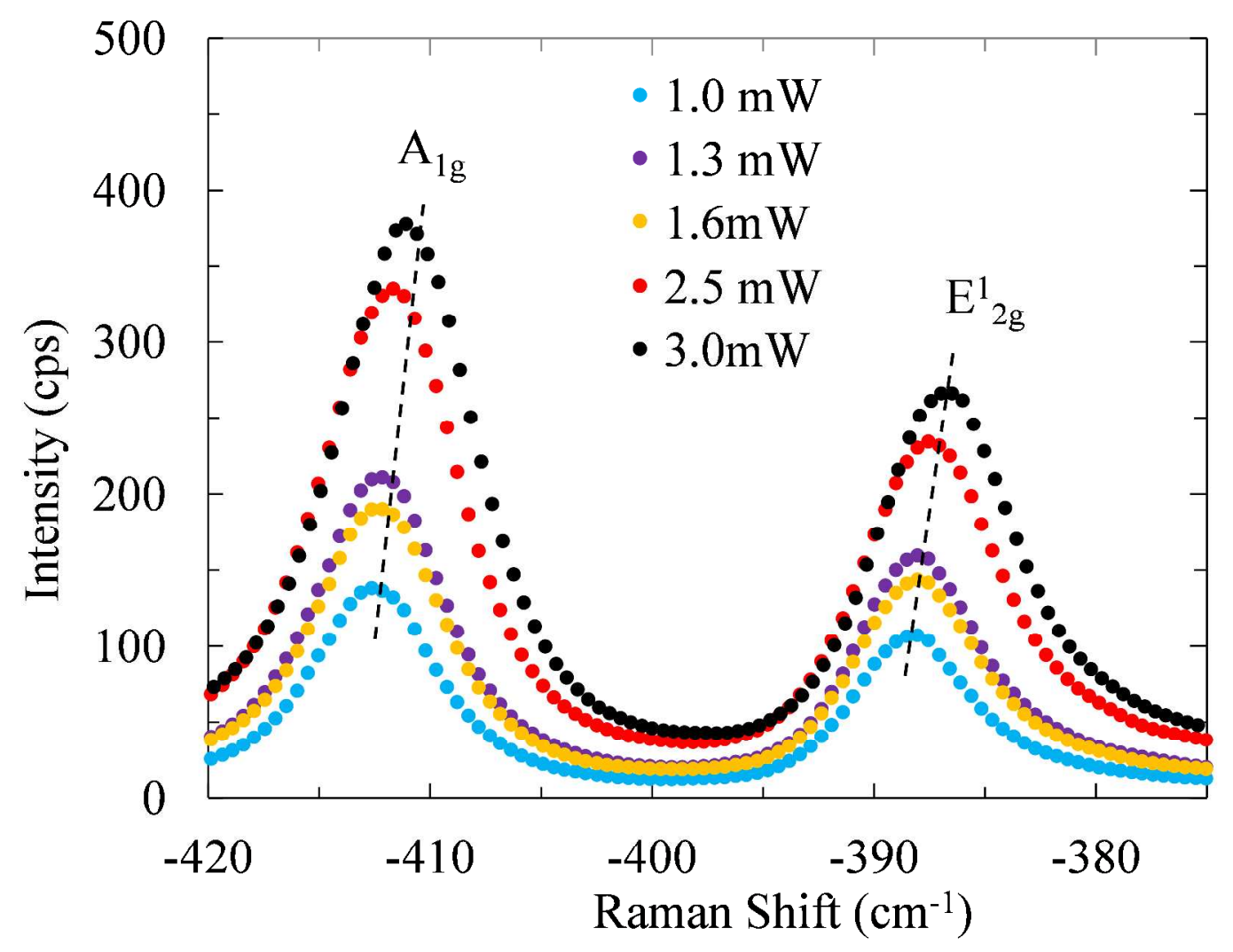

Figure S6: The anti-Stokes Raman shift of the trilayer $\mathrm{MoS}_{2}$-on-FP sample measure at different laser powers ranging from $1 \mathrm{~mW}$ to $3 \mathrm{~mW}$. The in-plane $\left(E_{2 g}^{1}\right)$ and out-of-plane $\left(A_{1 g}\right)$ characteristic resonances of the $\mathrm{MoS}_{2}$ film are marked on the plots. Dashed lines are guides to eye connecting peak positions of the $E_{2 g}^{1}$ and $A_{1 g}$ resonances at different laser powers. Peak positions of the $\mathrm{A}_{1 \mathrm{~g}}$ and $\mathrm{E}_{2 \mathrm{~g}}^{1}$ features manifest a linear red-shift by increasing the excitation laser power. 


\section{Calculation of the pre-factor $\kappa$ :}

$$
\kappa=\frac{\alpha_{I}+\alpha_{A S}}{\alpha_{I}+\alpha_{S}} \times\left(\frac{\lambda_{A S}}{\lambda_{S}}\right)^{3} \times \frac{S\left(\lambda_{I}, \lambda_{S}\right)}{S\left(\lambda_{I}, \lambda_{A S}\right)}
$$

Where $\alpha_{I}, \alpha_{S}$, and $\alpha_{A S}$ are absorption coefficients of the incident light $\left(\lambda_{I}\right)$, the $\mathrm{E}_{2 \mathrm{~g}}^{1}$ Stokes resonance $\left(\lambda_{S}\right)$, and the $\mathrm{E}_{2 \mathrm{~g}}^{1}$ anti-Stokes resonance $\left(\lambda_{A S}\right)$, respectively. Also, $S\left(\lambda_{I}, \lambda_{S}\right)$ and $S\left(\lambda_{I}, \lambda_{A S}\right)$ are the scattering cross-sections for scattering at $\lambda_{S}$ and,$\lambda_{A S}$ wavelengths, respectively, with excitation wavelength being $\lambda_{I}$. Table SI shows quantitative values used for $\mathrm{MoS}_{2}$ films in our modeling. In this table $\lambda_{S}$ and $\lambda_{A S}$ are calculated from Equation (S4), below, and different absorption coefficients are calculated from the imaginary part of the refractive index of the $\mathrm{MoS}_{2}$ as depicted in Figure S8.

TABLE SI:

\begin{tabular}{|c|c|c|c|c|c|c|}
\hline$\lambda_{I}(\mathrm{~nm})$ & $\lambda_{S}(\mathrm{~nm})$ & $\lambda_{A S}(\mathrm{~nm})$ & $\alpha_{I}\left(\mathrm{~cm}^{-1}\right)$ & $\alpha_{S}\left(\mathrm{~cm}^{-1}\right)$ & $\alpha_{A S}\left(\mathrm{~cm}^{-1}\right)$ & $\kappa$ \\
\hline 488 & 497 & 477 & $4.26 \times 10^{5}$ & $3.55 \times 10^{5}$ & $5.23 \times 10^{5}$ & 1.0878 \\
\hline
\end{tabular}




\section{Modeling of the light interaction with the trilayer $\mathrm{MoS}_{2}$ integrated on Fabry- Perot resonator (i.e., the $\mathrm{MoS}_{2}$-on-FP sample):}

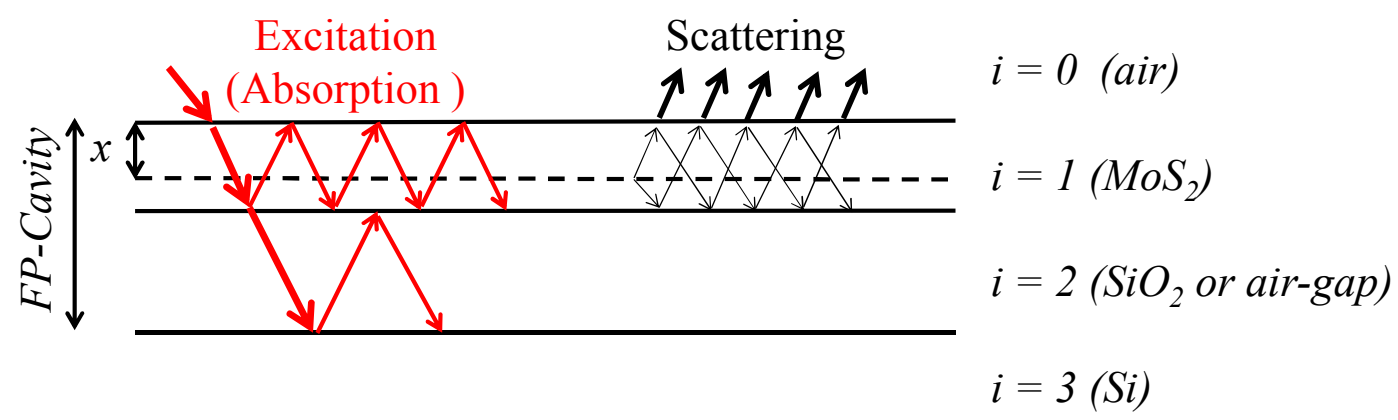

Figure S7: Schematic of the optical path of light in a FP cavity. Absorption (excitation) and scattering parts are shown separately. The depth " $x$ " in the $\mathrm{MoS}_{2}$ layer is highlighted with a dashed line. The trilayer $\mathrm{MoS}_{2}$ is shown with an exaggerated thickness for the sake of clarity.

Figure S7 illustrates the schematic of the light interference within a FP resonator (having either $\mathrm{SiO}_{2}$ or air-gap as the cavity), which is used to calculate the enhancement of the $\mathrm{E}_{2 \mathrm{~g}}^{1}$ Raman resonance intensity of the trilayer $\mathrm{MoS}_{2}$-on-FP sample. Here we have assigned an index $i=0,1$, 2, and 3 to air, $\mathrm{MoS}_{2}$ film, $\mathrm{SiO}_{2}$ or air-gap, and silicon (Si), respectively. For the sake of calculations, corresponding refractive indices, which are complex quantities in the most general form, are designated as $n_{i}$. Reflection and transmission coefficients of the electric field component of the light at $i-j$ boundaries (assuming normal incidence) are defined as $r_{i j}=\left(n_{i}-n_{j}\right)$ $/\left(n_{i}+n_{j}\right)$ and $t_{i j}=2 n_{i} /\left(n_{i}+n_{j}\right)$. Also, $\beta_{i}=2 \pi n_{i} d_{i} / \lambda$ and $\beta_{x}=2 \pi n_{1} x / \lambda$ are the amount of the phase change that light beam with wavelength $\lambda$ acquires over a path of length $d_{i}$ (thickness of $i$ th medium), and length $x$ in the trilayer $\mathrm{MoS}_{2}$ film (as shown in Figure S7), respectively. 
Following an approach discussed in Ref. [S2], we calculate total amplitude of the electric field components of the excitation (absorption) light (i.e., $E_{e x}$ or $E_{a b}$ ) at wavelength $\lambda$ and the corresponding $\mathrm{E}_{2 \mathrm{~g}}^{1}$ Raman scattered light $\left(\mathrm{E}_{\mathrm{sc}}\right)$ at depth $x$ of the $\mathrm{MoS}_{2}$ film as follows:

$$
E_{e x}=t_{01} \cdot \frac{e^{-i \beta_{x}}+R e^{-i\left(2 i \beta_{1}-\beta_{x}\right)}}{1+R r_{01} e^{-i 2 \beta_{x}}}
$$

and

$$
E_{S C}=t_{01} \cdot \frac{e^{-i \beta_{x}+R e^{-i\left(2 i \beta_{1}-\beta_{x}\right)}}}{1+R r_{01} e^{-i 2 \beta_{x}}},
$$

where, $R=\frac{r_{12}+r_{23} e^{-i 2 \beta_{2}}}{1+r_{12} r_{23} e^{-i 2 \beta_{2}}}$ is the effective reflection coefficient at the $\mathrm{MoS}_{2} / \mathrm{SiO}_{2}$ or $\mathrm{MoS}_{2} /$ airgap boundaries for a light of wavelength $\lambda$. Note that, although equations describing $E_{e x}$ (or $E_{a b}$ ) and $E_{s c}$ are similar, they work at different wavelengths of $\lambda_{e x}$ (or $\lambda_{a b}$ ) and $\lambda_{s c}$ that relate to each other as follows:

$$
\frac{1}{\lambda_{e x}}-\frac{1}{\lambda_{s c}}=E_{2 g}^{1} \text { resonance wavenumber }
$$

Thereby, the output intensity of the $\mathrm{E}_{2 \mathrm{~g}}^{1}$ Raman light is

$$
I_{E_{2 g}^{1}}=\int_{0}^{d_{1}}\left|E_{a b}(x) E_{s c}(x)\right|^{2} d x
$$

Therefore, based on Equation (S5), total enhancement factor (TEF) is defined as:

$$
\mathrm{TEF}=I_{E_{2 g}^{1}}\left(\mathrm{MoS}_{2}-\mathrm{on}-\mathrm{FP}\right) / I_{E_{2 g}^{1}}\left(\mathrm{MoS}_{2}-\mathrm{on}-\mathrm{Si}\right)
$$

Note that the wavelength-dependent refractive index of the trilayer $\mathrm{MoS}_{2}$ film, used in the modeling, over a wavelength range of 390-900 $\mathrm{nm}$ is shown in Figure S8. 


\section{Investigating separate contributions of the excitation (absorption) and scattering enhancement:}

Based on above discussions and the schematic of Figure S7, light absorption (A) and light scattering (S) by the trilayer $\mathrm{MoS}_{2}$ film can be computed as:

$$
\mathrm{A}=\int_{0}^{d_{1}}\left|E_{a b}(x)\right|^{2} d x
$$

and

$$
\mathrm{S}=\int_{0}^{d_{1}}\left|E_{s c}(x)\right|^{2} d x,
$$

where $E_{a b}$ and $E_{s c}$ are the electric field amplitudes of the light inside the $\mathrm{MoS}_{2}$ film and the scattered light outside the film, respectively. Therefore, absorption enhancement factor (AEF) and scattering enhancement factor (SEF) are defined as:

$$
\begin{aligned}
& \mathrm{AEF}=\mathrm{A}\left(\mathrm{MoS}_{2} \text {-on-FP) } / \mathrm{A}\left(\mathrm{MoS}_{2}\right. \text {-on-Si) }\right. \\
& \mathrm{SEF}=\mathrm{S}\left(\mathrm{MoS}_{2} \text {-on-FP }\right) / \mathrm{S}\left(\mathrm{MoS}_{2} \text {-on-Si }\right)
\end{aligned}
$$




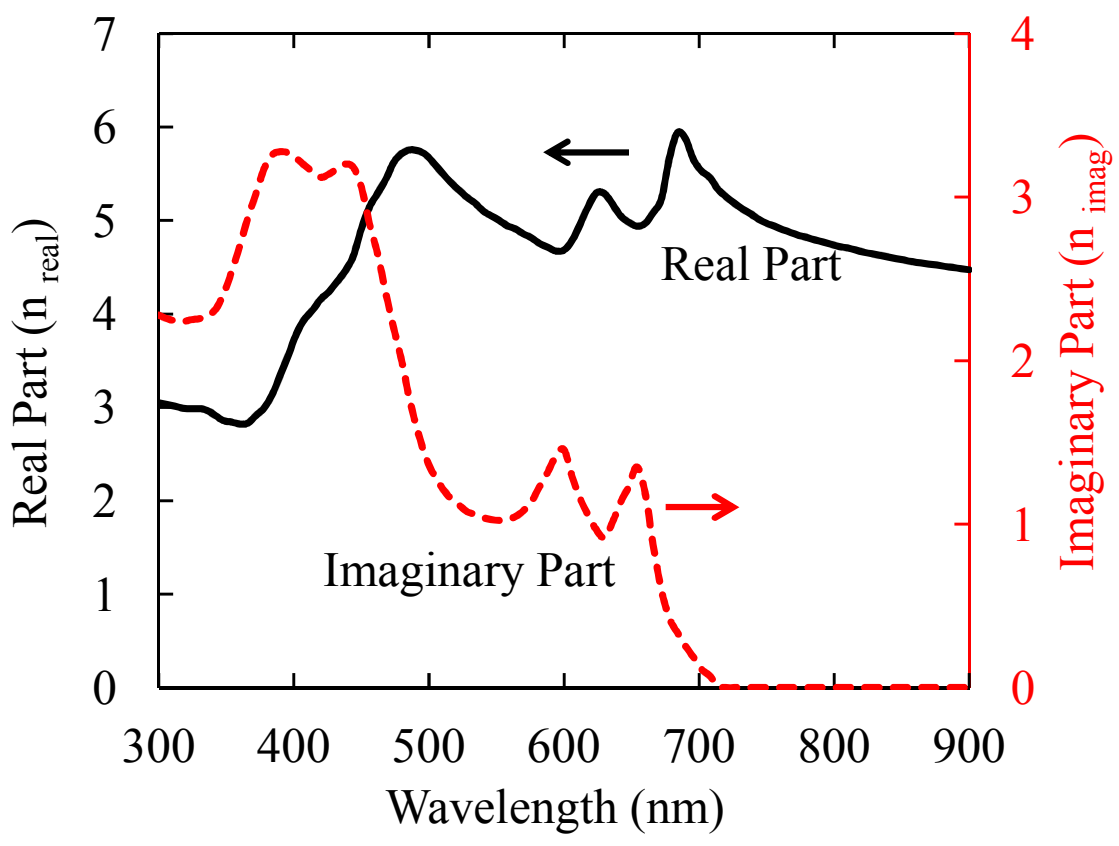

Figure S8: Real (left axis) and imaginary (right axis) parts of the refractive index of the $\mathrm{MoS}_{2}$ at different wavelengths used for the modeling of the light-MoS $\mathrm{M}_{2}$ interaction. 


\section{$\mathrm{MoS}_{2}$ thickness-dependent TEF:}
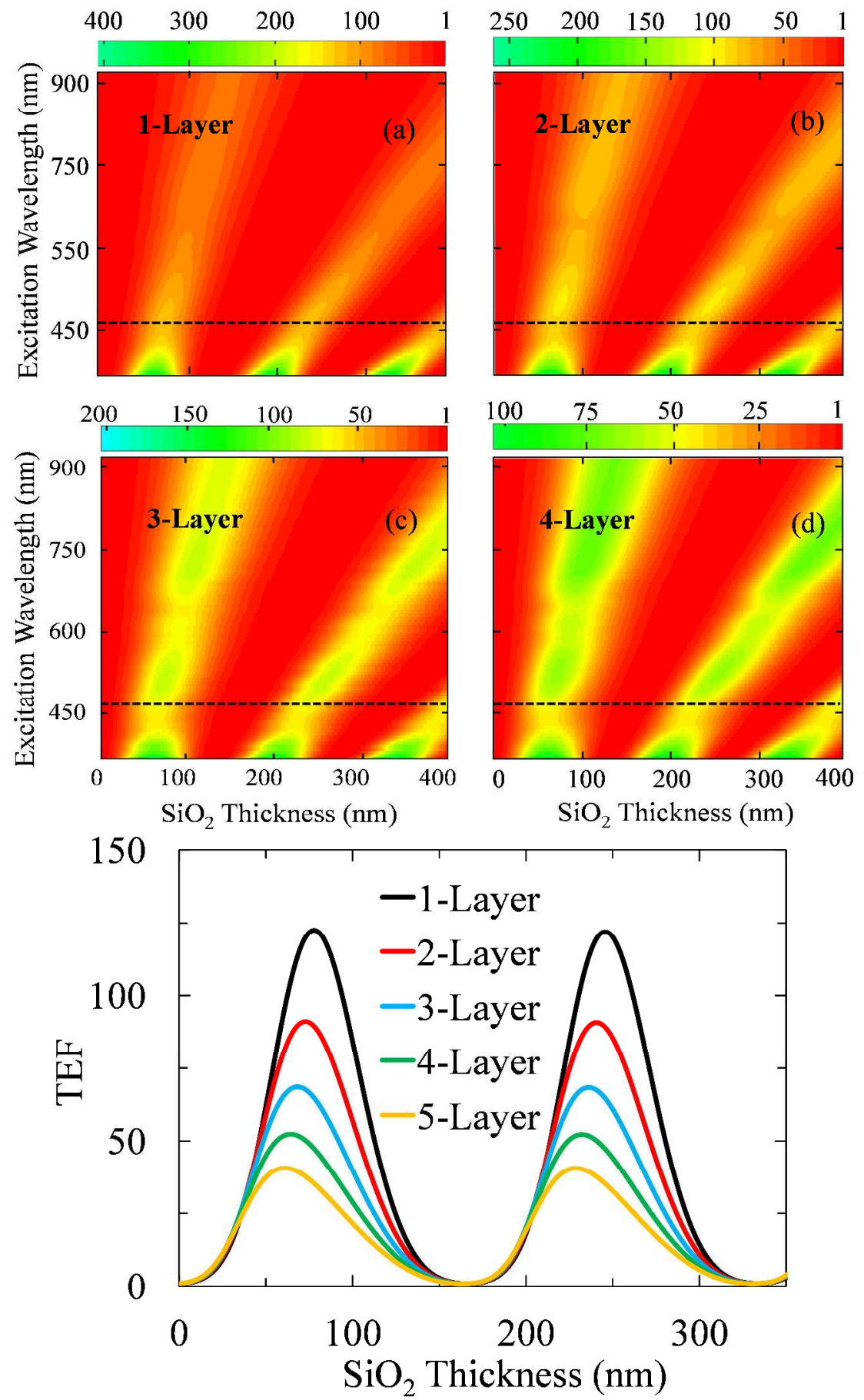

Figure S9: The $\mathrm{MoS}_{2}$ thickness-dependent TEF. The 2D map of TEF for (a) 1-layer, (b) 2-layer, (c) 3-layer, and (d) 4-layer $\mathrm{MoS}_{2}$ films. The color-bars represent the expected TEF induced by 
the FP resonance. (e) The comparison of TEFs that can be achieved for samples with $\mathrm{MoS}_{2}$ films of various thicknesses ranging from one to five layers when the samples are excited at $488 \mathrm{~nm}$ laser line (corresponding to the dashed lines overlaid on the 2D maps of (a)-(d)).

Two important observations can be made from Figure S9. First, increasing the number of $\mathrm{MoS}_{2}$ layers decreases the potential enhancement that can be achieved upon integration of the $\mathrm{MoS}_{2}$ film on the FP resonator. This effect can be explained on the basis of the large imaginary part of the refractive index of $\mathrm{MoS}_{2}$ at $488 \mathrm{~nm}(\sim 1.65)$, which causes optical loss and lowers the quality factor of the FP cavity. That is, in samples with thicker $\mathrm{MoS}_{2}$ films, light travels a longer path in the lossy part of the FP cavity and therefore, gains lower enhancement. Second, for thicker $\mathrm{MoS}_{2}$ samples, maximum TEF is achievable at a shorter FP cavity length. This fact can be explained based on the effective optical length (EOL) of the FP cavity as defined below:

$$
E O L=n_{\mathrm{SiO}_{2}} d_{\mathrm{SiO}_{2}}+n_{\mathrm{MoS}_{2}} d_{\mathrm{MoS}_{2}},
$$

where, ' $n$ ' and ' $d$ ' are, respectively, refractive index and thickness of the $\mathrm{SiO}_{2}$ and $\mathrm{MoS}_{2}$ layers. As discussed in the main text, the maximum enhancement can be reached when the effective optical length of the FP cavity is a quarter of the wavelength. Therefore, increasing the number of $\mathrm{MoS}_{2}$ layers (i.e., $d_{\mathrm{MoS}_{2}}$ in Eq. (S11)) decreases the required $\mathrm{SiO}_{2}$ thickness needed to satisfy the condition for maximum TEF. For instance, considering the refractive index of $\mathrm{MoS}_{2}$ at $\lambda=$ $488 \mathrm{~nm}$ to be 5.8 (Figure S8) and the thickness of an $\mathrm{MoS}_{2}$ individual layer to be $0.8 \mathrm{~nm}$ [S2], the optical length in the $\mathrm{MoS}_{2}$ film increases from $4.6 \mathrm{~nm}$ in the 1-layer sample (i.e., $5.8 \times(1 \times 0.8))$ to $23.2 \mathrm{~nm}$ in the 5-layer sample (i.e., $5.8 \times(5 \times 0.8))$. Thus, based on Eq. (S11), this change is balanced by the decrease in the $\mathrm{SiO}_{2}$ thickness. Figure $\mathrm{S} 9$ (e) shows that the decrease 
in the $\mathrm{SiO}_{2}$ thickness (at maximum TEF) is equal to the increase in the optical length imposed by increasing the number of $\mathrm{MoS}_{2}$ layers.

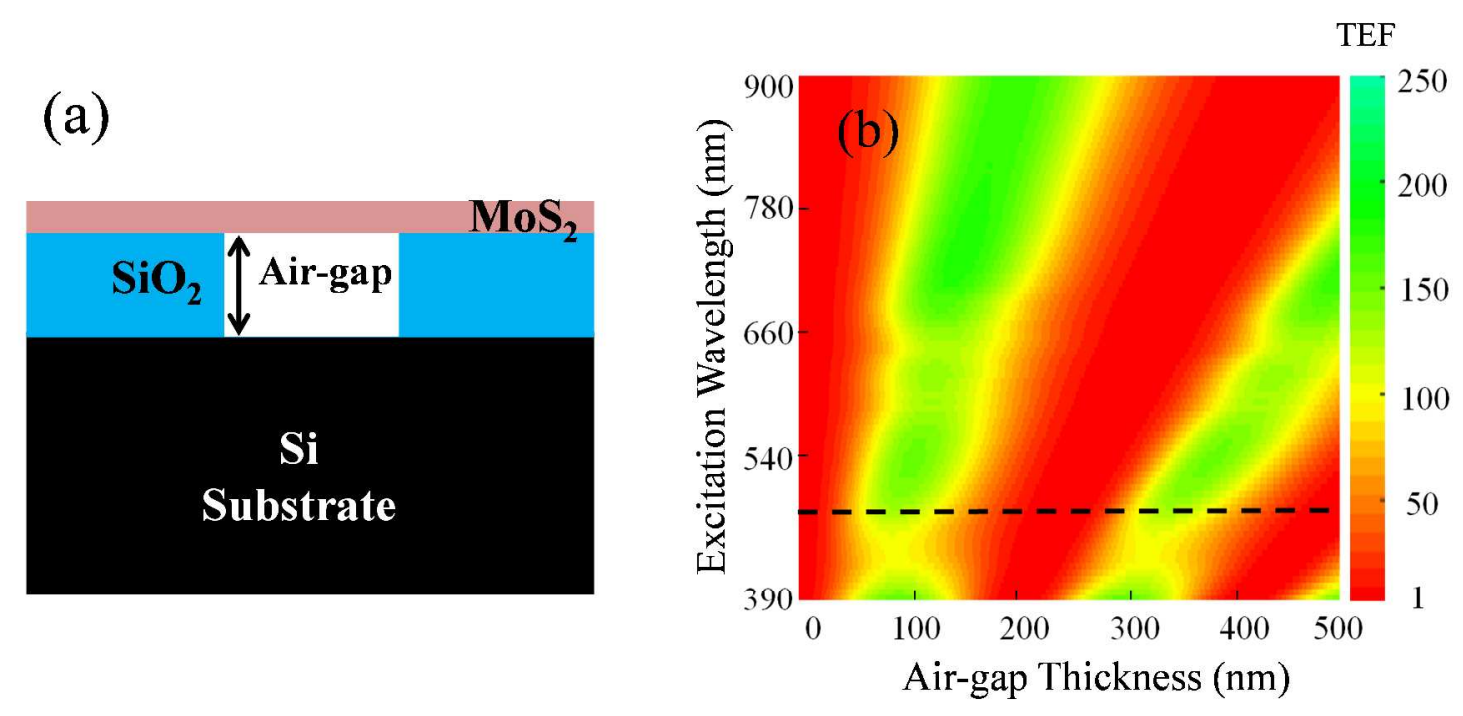

Figure S10: Enhanced light- $\mathrm{MoS}_{2}$ interaction induced by constructive interferences on the airgap FP resonator (studied for $\mathrm{E}_{2 \mathrm{~g}}^{1}$ Raman resonance). (a) Schematic showing structure of the airgap FP resonator fabricated through suspending trilayer $\mathrm{MoS}_{2}$ film over a hole etched into the $\mathrm{SiO}_{2}$ slab of $256 \mathrm{~nm}$ thick. The arrow depicts the thickness of the air-gap (cavity length). (b) Simulation results representing enhancement of Raman signal of the $\mathrm{MoS}_{2}$-on-FP sample as a function of the air-gap thickness (FP cavity length) and the wavelength of the excitation laser. The color-bar represents the expected TEF induced by the FP resonance. The dashed line locates the $488 \mathrm{~nm}$ excitation wavelength, which is used for analysis of the Figure 4 in the main text. 


\section{A note on the role of the cavity quality factor on light-MoS $\mathrm{S}_{2}$ interaction:}

The strength of the light interaction with $\mathrm{MoS}_{2}$ is proportional to $\mathrm{Q} / \mathrm{V}$, where $\mathrm{Q}$ is the quality factor of the cavity and $\mathrm{V}$ is the mode volume. That is, higher quality factor and smaller mode volume strengthen the interaction of light with the $\mathrm{MoS}_{2}$ layer and potentially lead to an excess heat generation. In our study, the mode volume is fairly large which is inherent to the FP cavity structure. However, there are several ways to change the quality factor of the resonators, among which changing reflection coefficients at two boundaries of cavity (i.e., upper and lower boundaries) is the most effective way. This can be accomplished by changing refractive index of the resonance media which through Fresnel's law leads to change in reflection coefficients at the boundaries of the cavity. Therefore, the plots shown in Figure 4 of the manuscript are actually a study of the impact of Q-factor on the strength of the light-MoS $\mathrm{M}_{2}$ interaction. As we discussed in the manuscript, changing resonance medium from $\mathrm{SiO}_{2}$ to air-gap increases Q-factor of the cavity and makes more light absorption possible (as shown in Figure 4f).

Another approach that we have taken to explore the impact of the Q-factor on the strength of the light-MoS $\mathrm{M}_{2}$ interaction is the study of the thickness dependent-TEF. As discussed in Figure S9, we showed that, increasing the number of the $\mathrm{MoS}_{2}$ layers adds extra loss to the cavity because of the large imaginary part of the $\mathrm{MoS}_{2}$. Thus, a reduction in the Q-factor of the cavity is expected as the number of the $\mathrm{MoS}_{2}$ layers increases. As a result of the lower Q factor, weaker interaction of the light with $\mathrm{MoS}_{2}$ and lower TEF are resulted for thicker $\mathrm{MoS}_{2}$ samples (Figure S9 (e)). 


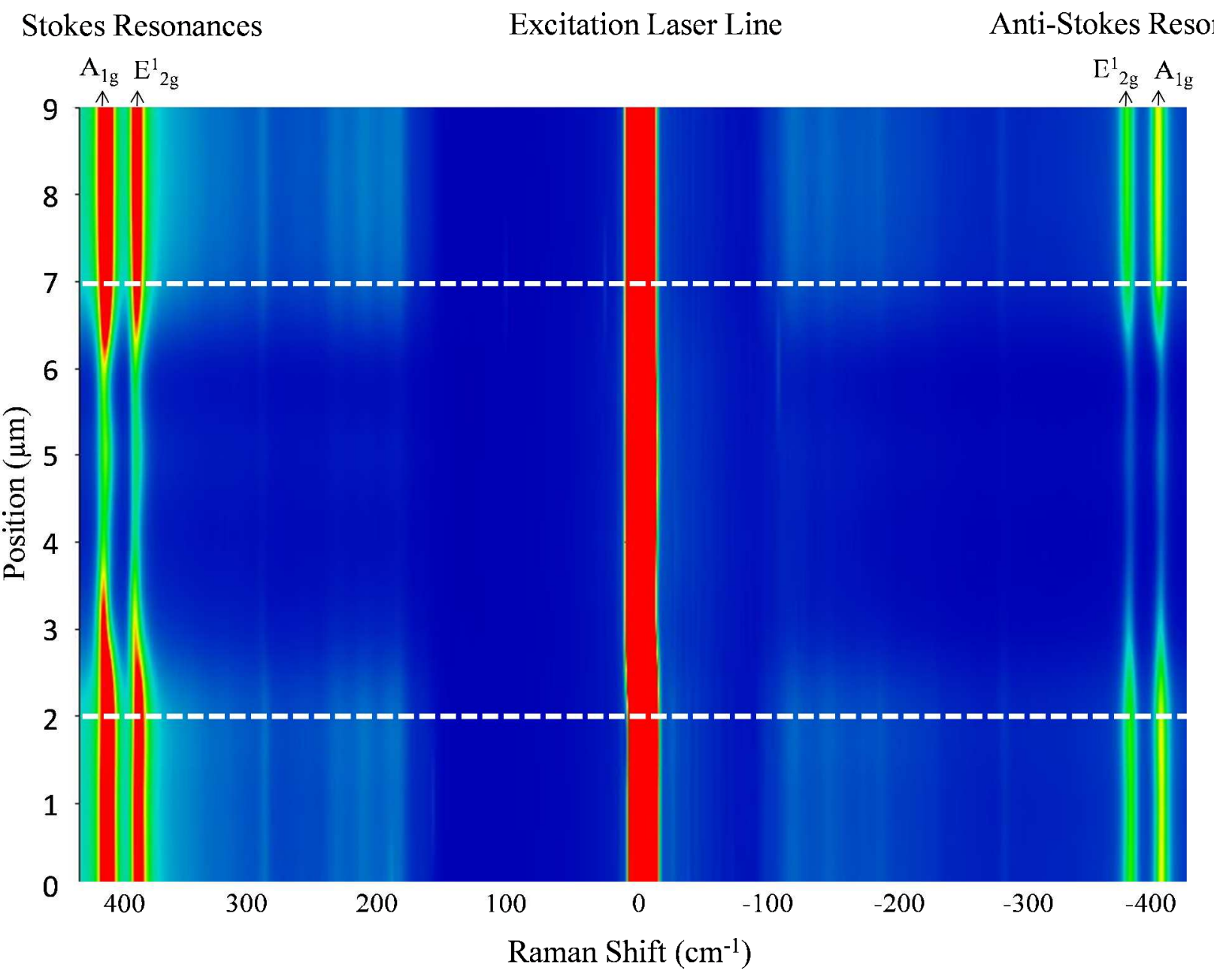

Figure S11: Line scan of the Stokes and anti-Stokes resonances of the trilayer $\mathrm{MoS}_{2}$ film across a hole of $5 \mu \mathrm{m}$ diameter, where $\mathrm{MoS}_{2}$ is suspended (as shown in Figure S10 (a)). The horizontal dashed lines show boundaries of the hole. The feature at wavenumber of $0 \mathrm{~cm}^{-1}$ is the excitation laser line.

\section{REFERENCES:}

[S1] X. Zhang, X.-F. Qiao, W. Shi , J.-B. Wu, D.-S. Jiang, and P.-H. Tan "Phonon and Raman Scattering of Two-Dimensional Transition Metal Dichalcogenides from Monolayer, Multilayer to Bulk Material" Chem. Soc. Rev., 2015, 44, 2757.

[S2] S. L. Li, H. Miyazaki, H. Song, H. Kuramochi, S. Nakaharai, and K. Tsukagoshi, "Quantitative Raman Spectrum and Reliable Thickness Identification for Atomic Layers on Insulating Substrates,” ACS Nano, vol. 6, no. 8, pp. 7381-7388, 2012. 